\title{
Morphological Filter-Assisted Ensemble Empirical Mode Decomposition
}

\author{
Xiaohang Zhou (D), Deshan Shan (D), and Qiao Li (iD) \\ Bridge Engineering Department, Southwest Jiaotong University, Chengdu 610031, China \\ Correspondence should be addressed to Deshan Shan; dsshan@swjtu.edu.cn
}

Received 23 April 2018; Revised 29 July 2018; Accepted 7 August 2018; Published 17 September 2018

Academic Editor: Alessandro Della Corte

Copyright (c) 2018 Xiaohang Zhou et al. This is an open access article distributed under the Creative Commons Attribution License, which permits unrestricted use, distribution, and reproduction in any medium, provided the original work is properly cited.

\begin{abstract}
In the ensemble empirical mode decomposition (EEMD) algorithm, different realizations of white noise are added to the original signal as dyadic filter banks to overcome the mode mixing problems of empirical mode decomposition (EMD). However, not all the components in white noise are necessary, and the superfluous components will introduce additional mode mixing problems. To address this problem, morphological filter-assisted ensemble empirical mode decomposition (MF-EEMD) was proposed in this paper. First, a new method for determining the structuring element shape and size was proposed to improve the adaptive ability of morphological filter (MF). Then, the adaptive MF was introduced into EMD to remove the superfluous white noise components to improve the decomposition results. Based on the contributions of MF in a single EMD process, the MF-EEMD was proposed by combining EEMD with MF to suppress the mode mixing problems. Finally, an analog signal and a measured signal were used to verify the feasibility of MF-EEMD. The results show that MF-EEMD significantly mitigates the mode mixing problems and achieves a higher decomposition efficiency compared to that of EEMD.
\end{abstract}

\section{Introduction}

Signal decomposition techniques, such as Fourier transform (FT), wavelet transform (WT), and empirical mode decomposition (EMD), are widely used in the field of structural health monitoring for denoising, parameter identification, and damage detection [1-3]. However, these techniques have considerable deficiencies. FT [4] takes the signal as a linear combination of sine waves with different frequencies, but the frequency and amplitude of the sine wave are immutable. Consequently, FT cannot analyze nonstationary or nonlinear signals [5]. Short-time Fourier transform (STFT) is a variation of FT, and it can address nonstationary signals by employing the window function technique. However, it is hard to balance the resolution between the time domain and the frequency domain [6]. WT [7] is suitable for decomposing nonlinear signals with multiple scales and multiple resolutions, but determining the appropriate mother wavelet function is difficult. Hence, the adaptive performance of WT is poor. EMD is a fully data-driven technique with the ability to decompose nonstationary and nonlinear signals; thus, it possesses strong robustness and adaptive ability [5]. However, the mode mixing problems in EMD severely restrict its application $[8,9]$.

To overcome the mode mixing problem of EMD, Wu and Huang [10] proposed the noise-assisted ensemble empirical mode decomposition (EEMD). In the EEMD algorithm, EMD is performed repeatedly with the addition of different white noises, and an ensemble of the same order intrinsic mode functions (IMF) is made to obtain the final decomposition results. Flandrin and Rilling [11] noted that the white noise added in EEMD could be taken as a set of dyadic filter banks, and different frequency components in the original signal were projected to the corresponding banks. This characteristic of white noise helped to separate the different orders of IMFs.

The valuable components in the original signal are typically limited in quantity; in other words, the number of essential dyadic filter banks for EEMD can be counted. However, the added white noise contains all the dyadic filter banks in the frequency domain. The superfluous dyadic filter banks added to the original signal will not only reduce the 


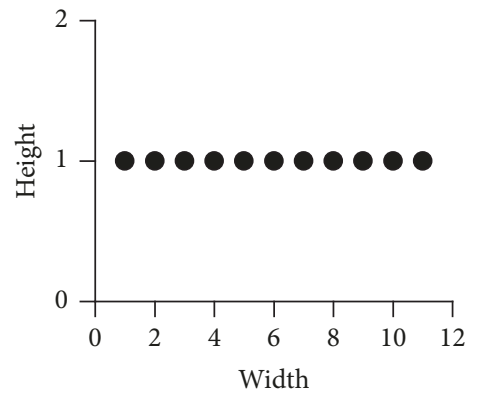

(a) Linear

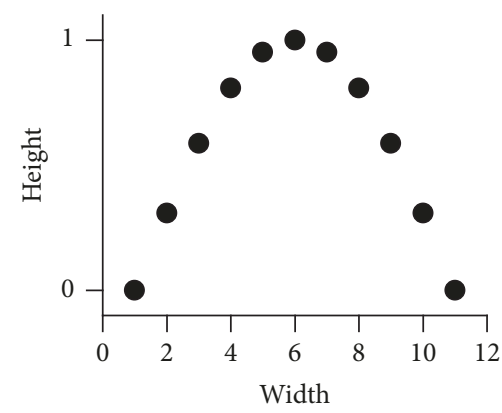

(b) Sinusoidal

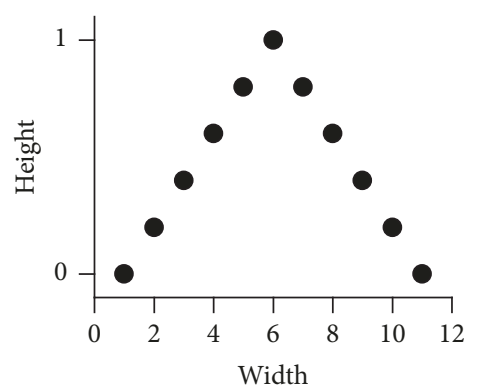

(c) Triangular

FIGURE 1: Different shapes of structuring elements.

decomposition efficiency but also cause additional mode mixing problems [12]. Based on the above problems, it could be speculated that the accuracy and efficiency of EEMD would be improved if the superfluous dyadic filter banks were removed.

The morphological filter (MF) is a type of nonlinear filter that is particularly sensitive to impulse noise and white noise; moreover, it will not cause sudden truncation in the frequency domain or phase delay in the time domain $[13,14]$. Deng [15] adopted the MF along with other traditional filters to filter impulse noise, and the results demonstrated that the MF was superior to the other filters, especially when the noise level was high. Yuan [16] adopted the MF and a hybrid filter to detect and eliminate impulse noise in images, and superb denoising results were achieved for different signal-tonoise ratios. Lin [17] used the MF to eliminate the high-ratio impulse noise, and the MF achieved a better performance than other traditional digital filters, such as the mean filter and the median filter. Saniie [14] adopted the MF to suppress the impulse noise and the white noise in an ultrasonic signal; the MF could significantly improve the signal-to-noise ratio and maintain an undistorted signal. Mukhopadhyay [18] adopted the MF to filter different types of noise, and the results showed that the MF was effective for all types of noise and superior to other standard noise removal algorithms. The above literature review illustrates that the MF is a potentially powerful tool for filtering superfluous dyadic filter banks in EEMD.

This paper proposes the morphological filter-assisted ensemble empirical mode decomposition (MF-EEMD) to overcome the mode mixing problems in EEMD. First, the concepts of MF were presented, and the adaptive ability of MF was improved by proposing a new method for determining the structure element shape and size. Then, the adaptive MF was applied to remove the superfluous white noise components in EMD to improve its decomposition results. Based on the contributions of MF in a single EMD process, the MFEEMD was proposed by introducing MF into EEMD to suppress its mode mixing problems. Finally, MF-EEMD is compared with EEMD by decomposing an analog signal and a measured signal, and the effectiveness of MF-EEMD is validated.

\section{MF Theory}

In 1986, Serra $[19,20]$ proposed the MF based on the theory of mathematical morphology. The MF is a type of nonlinear temporal filter that can process a signal directly in the time domain without converting it into the frequency domain. In the MF algorithm, a structuring element is used to address the data points, and its common shapes are shown in Figure 1.

In the algorithm of MF, dilation and erosion are the two most basic operations [21]. The dilation operation on signal $\alpha$ with structuring element $\beta$ is defined as $\alpha \oplus \beta$, and the erosion operation on signal $\alpha$ with structuring element $\beta$ is defined as $\alpha \Theta \beta$, as shown in (1) and (2), respectively.

$$
\begin{aligned}
& \alpha \oplus \beta=\max \{\alpha(i-j)+\beta(j)\} \\
& \alpha \oplus \beta=\min \{\alpha(i+j)-\beta(j)\}
\end{aligned}
$$

where $1<i<n, 1 \leq j \leq k, i+j \leq n, n$ is the length of $\alpha$, and $k$ is the width of $\beta$.

Based on the dilation and erosion operations, the opening operation on signal $\alpha$ with structuring element $\beta$ is defined as $\alpha \circ \beta$, and the closing operation on signal $\alpha$ with structuring element $\beta$ is defined as $\alpha \cdot \beta$, as shown in (3) and (4), respectively.

$$
\begin{aligned}
& \alpha \circ \beta=(\alpha \oplus \beta) \oplus \beta \\
& \alpha \bullet \beta=(\alpha \oplus \beta) \oplus \beta
\end{aligned}
$$

However, the opening operation can only address the local maximums, while the closing operation can only address the local minimums [22]. To address both the local maximums and local minimums of the signal, the opening and closing operations should be combined by (5).

$$
\begin{aligned}
\alpha^{\prime} & =(\alpha \circ \beta) \bullet \beta \\
\text { or } \alpha^{\prime} & =(\alpha \bullet \beta) \circ \beta
\end{aligned}
$$

To eliminate the effect of different operational sequences, the average of opening-closing and closing-opening is taken as the final filtering result. The complete operational flow of the MF is shown in Figure 2. 


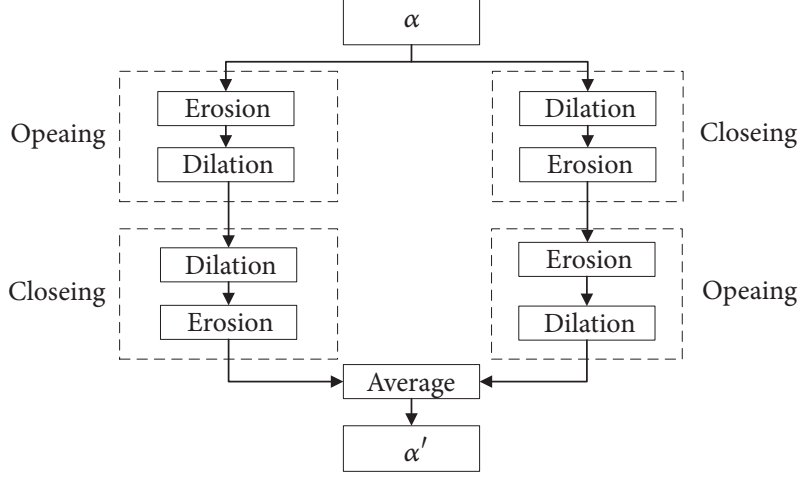

FIGURE 2: Operational flow of the MF.

\section{Adaptive MF}

The shape and size of the structuring element are the two most important parameters in MF, and they have significant influences on the filtering results. The determination of the two parameters relates to multiple factors, such as the sample frequency, the dominant frequency, and the noise type. The shape and size of the structuring element are generally determined by empirical methods $[14,15]$, and the disadvantages of those methods are low adaptive ability and poor versatility.

To construct an adaptive MF algorithm, the determination method of the appropriate structuring element parameters were investigated in this paper.

A composite signal of sinusoid and white noise is constructed based on (6)-(8)

$$
\begin{aligned}
S_{s i n} & =\lambda \cdot \sin \left(2 \pi f \times \frac{n}{s f}\right) \\
S_{w n} & =k \cdot \operatorname{rand}\left(\frac{n}{s f}\right) \\
S_{c} & =S_{\text {sin }}+S_{w n}
\end{aligned}
$$

where $S_{s i n}$ is a pure sinusoid, $S_{w n}$ is white noise, $S_{c}$ is the composite signal, $s f$ is the sample frequency, $n=1,2,3 \ldots N$, $N$ is the length of $S_{c}, f$ is the dominant frequency, $\lambda$ is the amplitude of the pure sinusoid, and $k$ is the amplitude of the white noise.

To make an objective evaluation of the effectiveness of the MF, the signal-to-noise ratio (SNR) [23] is introduced as the evaluation index, as shown in (9).

$$
S N R=10 \times \log \left(\frac{\operatorname{Std}\left(S_{\text {sin }}\right)}{\operatorname{Std}\left(S_{M F}-S_{\text {sin }}\right)}\right)
$$

where the operator $\operatorname{Std}()$ is the expression of standard deviation and $S_{M F}$ is the filtering result of $S_{c}$. When $S_{M F}$ and $S_{s i n}$ are more similar, the value of $S N R$ is greater.

3.1. Shape Selection. To investigate the characteristics of different structuring element shapes, a composite signal with one dominant frequency was constructed by (6)-(8) with the parameters $s f=256 \mathrm{~Hz}, N=512, f=5 \mathrm{~Hz}, \lambda=1$, and $k=0.3$, as shown in Figure 3.
TABLE 1: Parameter settings of $S_{c}$ with one dominant frequency.

\begin{tabular}{lcccc}
\hline No. & $s f$ & $f$ & $\lambda$ & $k$ \\
\hline$\# 1$ & 256 & 5 & 1 & 0.1 \\
$\# 2$ & 256 & 5 & 1 & 0.3 \\
$\# 3$ & 256 & 5 & 1 & 0.5 \\
$\# 4$ & 512 & 4 & 1 & 0.3 \\
$\# 5$ & 512 & 8 & 1 & 0.3 \\
$\# 6$ & 512 & 16 & 1 & 0.3 \\
$\# 7$ & 64 & 3 & 1 & 0.3 \\
$\# 8$ & 128 & 3 & 1 & 0.3 \\
$\# 9$ & 512 & 3 & 1 & 0.3 \\
\hline
\end{tabular}

Three types of structuring elements were constructed, each with linear, sinusoidal, or triangular shapes. The range of the structuring element width $(W)$ was set as 3-35, and the range of the structuring element height $(H)$ was set as $0.1-$ 5 times the standard deviation of $S_{c}$. Then, the three types of structuring elements with varying sizes were adopted to filter the signal, and the corresponding $S N R$ of each $S_{M F}$ was calculated. In Figure 4, the initial $S N R$ of $S_{c}$ is shown as a plane surface with a value of 14.35, and the $S N R$ of $S_{M F}$ is shown as a curved surface.

In Figure 4(a), the changes in SNR values only relate to $W$. The appropriate distribution range of $W$ is 3-11, and the best filtering result is achieved with an $S N R$ value of 19.56 when $W=5$. In Figure 4(b), the changes in $S N R$ values relate to both $W$ and $H$. $W$ and $H$ have wide appropriate ranges, and the best filtering result is achieved with an $S N R$ value of 23.72 when $W=11$ and $H=0.7$. The filtering result in Figure 4(c) is similar to that in Figure 4(b), but the appropriate ranges of $W$ and $H$ are narrower. The best filtering result is achieved with an $S N R$ value of 22.61 when $W=9$ and $H=0.5$.

Although the filtering results with different white noises are not constant due to the white noise randomness, still some patterns can be observed as follows. The best filtering results of the sinusoidal and triangular structuring elements are similar, and they are superior to that of the linear element. Additionally, the distribution range of the appropriate element sizes of the triangular structuring element is narrower than that of the sinusoidal structuring element and, thus, the distribution law of the appropriate element size of the triangular structuring element is more obvious.

According to the above patterns, the triangular structuring element was selected for the follow-up studies in this paper.

3.2. Size Determination. To investigate the distribution laws of the appropriate $W$ and $H$, multiple composite signals with one dominant frequency were constructed using (6)-(8). The corresponding parameter settings are listed in Table 1. A triangular structuring element with varying sizes was adopted to filter the signals, and the corresponding $S N R$ values of each $S_{M F}$ were calculated, as shown in Figure 5.

In signals \#1-3, $k$ is the only parameter that is varied. The distribution range of appropriate sizes becomes wider with increasing $k$; however, the ridgeline (colored yellow) of the 


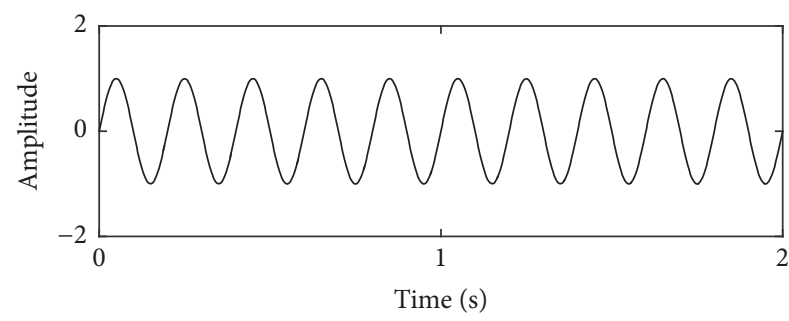

(a) $S_{\text {sin }}$

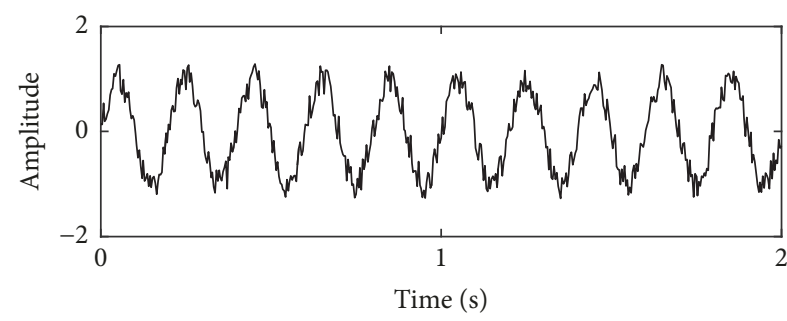

(b) $S_{c}$

FIgURE 3: Composite signal with one dominant frequency.

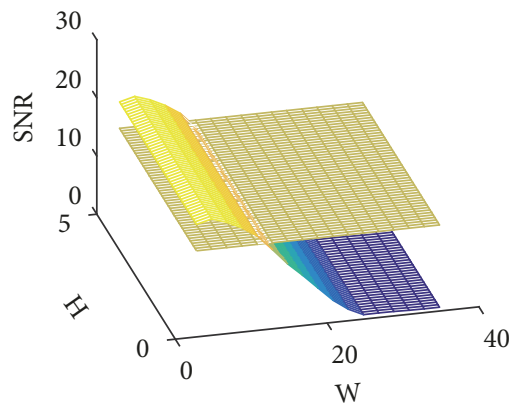

(a) Linear

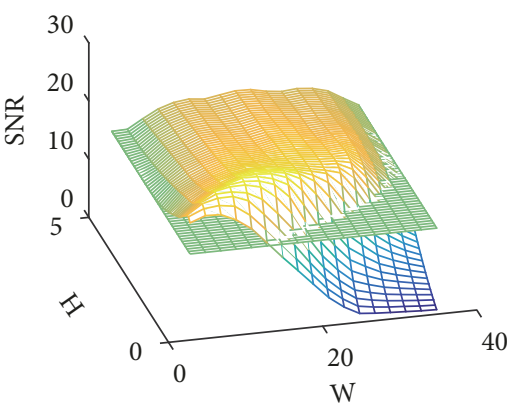

(b) Sinusoidal

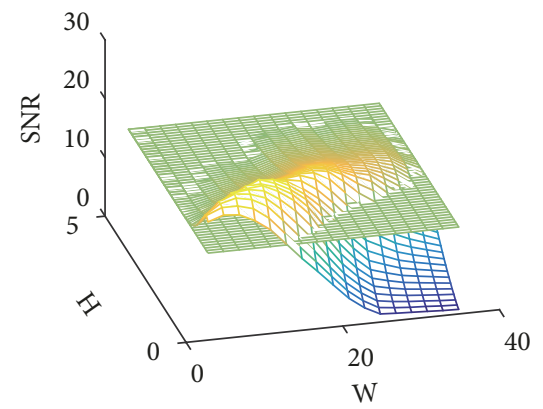

(c) Triangular

FIGURE 4: Filtering results with different structuring element shapes.

$S N R$ surface remains relatively unchanged. In signals \#4-6, $f$ is the only parameter being varied. When $f$ is small, the ridgeline is close to axis $W$, and vice versa. In signals \#7-9, sf is the only parameter being varied, and the effect of $s f$ on the ridgeline is opposite that of $f$.

Figure 5 illustrates that the location and direction of the ridgeline of $S N R$ surface are mainly dominated by $s f$ and $f$. The intersection point of the ridgeline and $H=0.1$ has a larger value with increases in $s f$ or decreases in $f$. The value of $W / H$ also has the same relationship with $s f$ and $f$. The above laws can be summarized as (10)-(12).

$$
\begin{aligned}
W_{H=0.1} & \backsim \frac{s f}{f} \\
W / H & \backsim \frac{s f}{f} \\
W & \backsim \frac{s f}{f}+H \cdot \frac{s f}{f}
\end{aligned}
$$

A linear regression was performed to fit (12) using 650 samples with the value of $s f / f$ varying from 4 to 1,024 . The fitting result is shown in (13).

$$
W=0.33 \sqrt{\frac{s f}{f}}+0.24 H \cdot \frac{s f}{f}
$$

In Figure 6, three examples with different values of $s f / f$ were selected to demonstrate the prediction precision of (13). The black points are the predictions of the appropriate structuring element sizes, and they are very close to the ridgelines
TABle 2: Parameter settings of $S_{c}$ with two dominant frequencies.

\begin{tabular}{lcccccc}
\hline No. & $s f$ & $f_{l}$ & $f_{h}$ & $\lambda_{l}$ & $\lambda_{h}$ & $k$ \\
\hline$\# 1$ & 256 & 8 & 16 & 0.4 & 0.6 & 0.3 \\
$\# 2$ & 256 & 2 & 8 & 0.5 & 0.5 & 0.3 \\
$\# 3$ & 256 & 4 & 32 & 0.6 & 0.4 & 0.3 \\
\hline
\end{tabular}

of the SNR surfaces, which indicates that the prediction of appropriate structuring element sizes has a high precision.

To investigate the applicability of (13) to signals with multiple dominant frequencies, three composite signals with two dominant frequencies were constructed with the parameter settings listed in Table 2. The SNR values of the filtering results along with the predictions of the appropriate structuring element sizes are shown in Figure 7, where the blue points represent the predictions with $f_{h}$ and the red points represent the predictions with $f_{l}$.

Figure 7 illustrates that the ridgeline of the SNR surface is between the two predictions but very close to the blue points, indicating that the distribution of appropriate structuring element sizes is mainly dominated by $f_{h}$. Therefore, the ridgeline of the $S N R$ surface can be approximately estimated by (13) with $f_{h}$.

In actual operation, one pair of appropriate size $(W, H)$ is sufficient for the MF, and the value of $W$ for the triangular structuring element is $W=2 n+1$, which means that the smallest value of $W$ is 3 . In this paper, a simplified and practical size determination method for the triangular structuring element was proposed as follows: (1) Extract the highest valuable frequency $f$ of the signal. (2) Introduce $s f$ and $f$ into (13), 

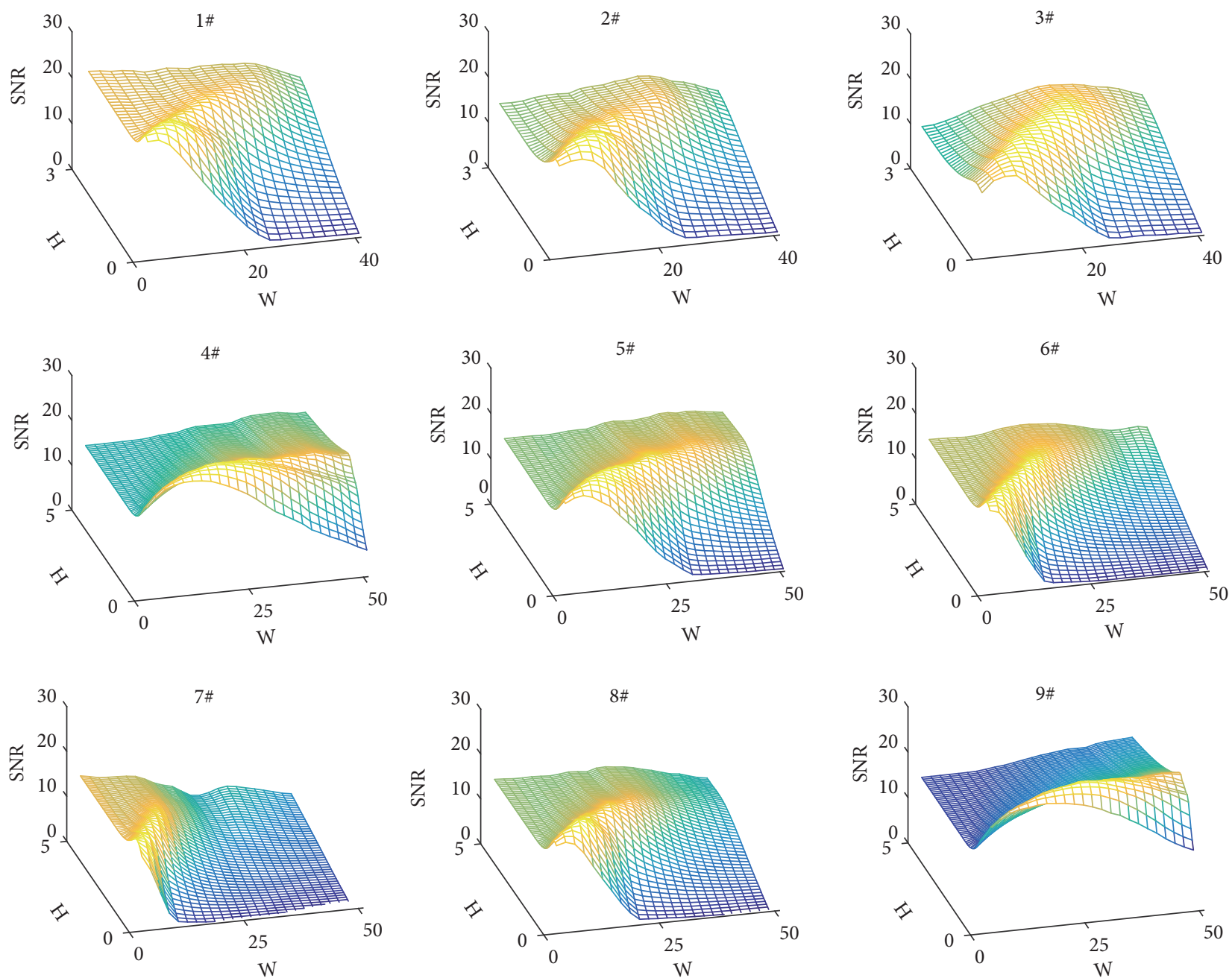

FIGURE 5: Filtering results with varying structuring element sizes.

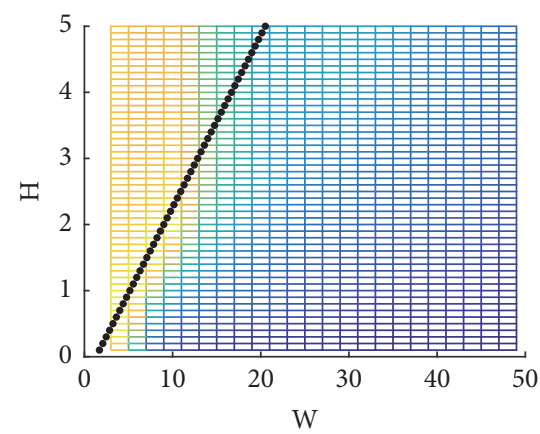

(a) $s f / f=16$

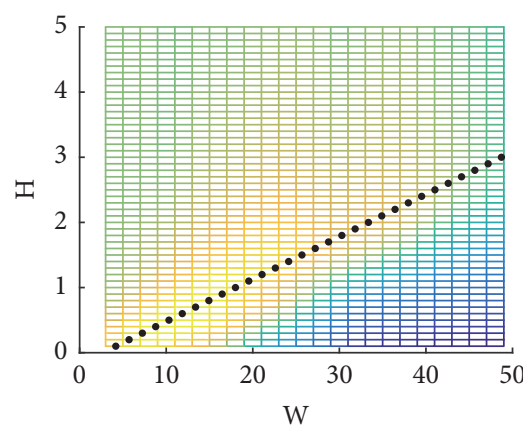

(b) $s f / f=64$

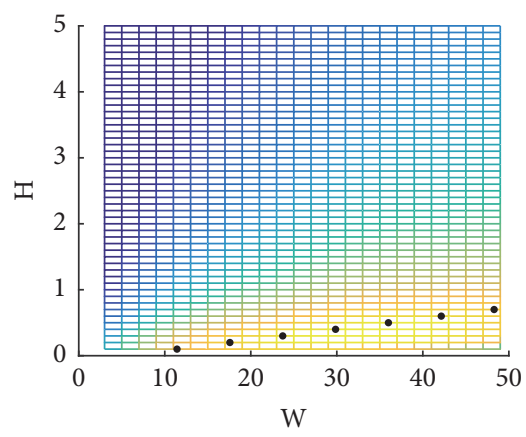

(c) $s f / f=256$

FIGURE 6: Fitting results of the SNR ridgeline with one dominant frequency. 


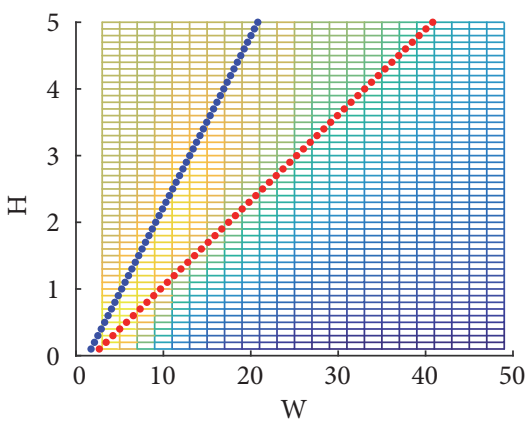

(a) $f_{l}=8, f_{h}=16$

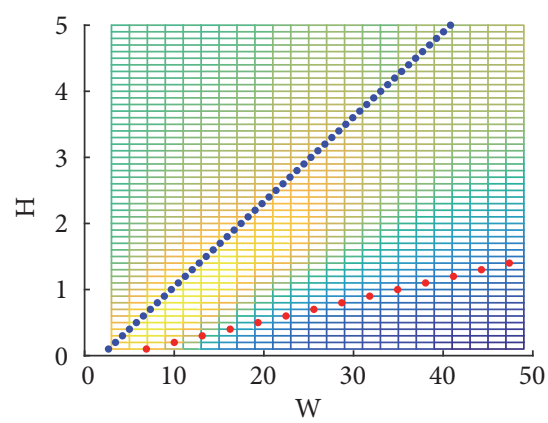

(b) $f_{l}=2, f_{h}=8$

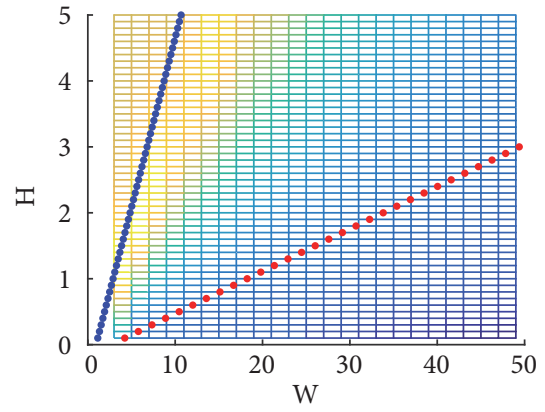

(c) $f_{l}=4, f_{h}=32$

FIGURE 7: Fitting results of the SNR ridgeline with two dominant frequencies.

increase $H$ from 0.1 with a step length of 0.1 , and calculate the corresponding odd-value of $W$. (3) Stop the calculation when $W \geq 3$, and obtain one pair of appropriate size $(W, H)$.

\section{MF-EEMD}

The superfluous white noise components in EEMD may cause additional mode mixing problems and reduce the decomposition efficiency [12]. In this paper, the MF was adopted to filter out the superfluous white noise components to improve EEMD.

4.1. EMD Combined with MF. A composite signal with two dominant frequencies was constructed by (6) -(8), as shown in Figure 8 , and the parameters were set as $f_{1}=2, \lambda_{1}=0.5, f_{2}=5$, $\lambda_{2}=0.5, s f=256, N=512$, and $k=0.3$.

EMD was directly adopted for the composite signal in Figure 8(c). The stoppage criterion of EMD was that the number of zero crossing points of IMF must be larger than $N / s f$, and the decomposition shall be stopped after the appearance of the first unqualified IMF. This stoppage criterion was also applicable to EEMD and MF-EEMD. There were 9 orders of IMFs in the decomposition results of EMD, as shown in Figure 9. The $1^{\text {st }}$ - to $3^{\text {rd }}$-order IMFs are noise, the $4^{\text {th }}$-order IMF consists of noise and a $5 \mathrm{~Hz}$ component, the $5^{\text {th }}$-order IMF consists of $5 \mathrm{~Hz}$ and $2 \mathrm{~Hz}$ components, the $6^{\text {th }}$-order IMF is mainly a $2 \mathrm{~Hz}$ component, and the $7^{\text {th }}$ - to $9^{\text {th }}$-order IMFs are trends. These results illustrate that there are obvious mode mixing problems in the EMD results.

There is typically no prior knowledge about the highest valuable $f$ in the signal; thus, the application of (13) is limited. To address this problem, an extraction method for $f$ based on the decomposition results of EMD or EEMD was proposed.

First, the original signal is decomposed with EMD or EEMD. Then, the criterion ET proposed by $\mathrm{Wu}$ and Huang [24] is used to distinguish the noise components from the valuable components. The criterion $E_{n} T_{n}$ of the $n^{\text {th }}$ IMF is defined by (14) and (15).

$$
\begin{aligned}
& E_{n}=\frac{1}{N} \sum_{i=1}^{N}\left[\operatorname{IMF}_{n}(i)\right]^{2} \\
& T_{n}=\frac{2 N}{N_{z c}}
\end{aligned}
$$

where $E_{n}$ is the energy density, $T_{n}$ is the averaged period, $N$ is the length of $\mathrm{IMF}_{n}$, and $N_{z c}$ is the number of zero-crossings of $\mathrm{IMF}_{n}$. When $\mathrm{IMF}_{n}$ is a noise component, the value of $E_{n} T_{n}$ is small; otherwise, the value of $E_{n} T_{n}$ is considerably larger.

By observing the values of $E_{n} T_{n}$, the noise components and valuable components are distinguished, and the IMF with the desired $f$ is selected. Finally, the auto-power spectrum analysis is adopted for the selected IMF, and the frequency of the peak point in the spectrum is taken as $f$.

The ET values of the IMFs in Figure 9 are shown in Figure 10 , where there is a sudden increase between the $3^{\text {rd }}$ and $4^{\text {th }} E T$ values. This result indicates that the frequency of the $4^{\text {th }}$ IMF can be taken as $f$. The auto-power spectrum of the $4^{\text {th }}$ IMF is shown in Figure 11 with $f=5 \mathrm{~Hz}$.

The appropriate structuring element size was derived as $W=3$ and $H=0.1$ by (13) with $s f=256$ and $f=5$. Then, the composite signal in Figure 8(c) was filtered by the MF, as shown in Figure 12.

The Fourier spectrum of the noise component is shown in Figure 13. The amplitude of the low-frequency domain, where the frequencies of the valuable components are located, is smaller than that of the high-frequency domain. In other words, the superfluous dyadic filter banks are removed while the valuable ones are retained in $S_{M F}$.

Then, the EMD was applied again to the filtering result in Figure 12(a), and 7 orders of IMFs were obtained, as shown in Figure 14. The $1^{\text {st }}$ - to $3^{\text {rd }}$-order IMFs are noise, the $4^{\text {th }}$-order IMF is a $5 \mathrm{~Hz}$ component, the $5^{\text {th }}$-order IMF is mainly a $2 \mathrm{~Hz}$ component, and the $6^{\text {th }}$ - and $7^{\text {th }}$-order IMFs are trends. Only slight mode mixing problems are observed in the decomposition results, indicating that the mode mixing problems are suppressed. Moreover, the number of IMFs is reduced from 9 to 7, which means that the decomposition efficiency of EMD is also improved.

4.2. EEMD Combined with MF. The decomposition results of a single operation of EMD were improved with the addition of MF. EEMD is an ensemble of numerous EMD; therefore, the EEMD results would also be improved by the MF. Hence, MF-EEMD was proposed in this paper by introducing MF into EEMD. 


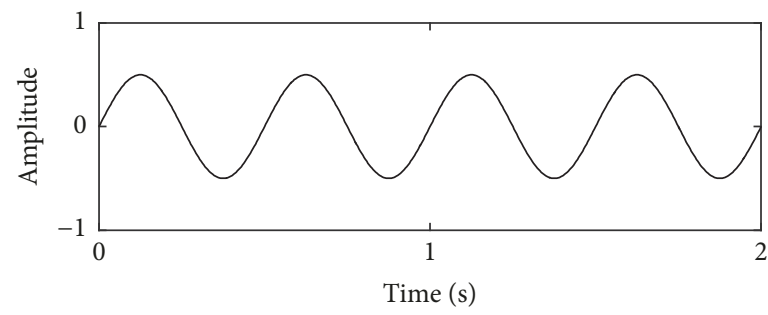

(a) $S_{\sin 1}$

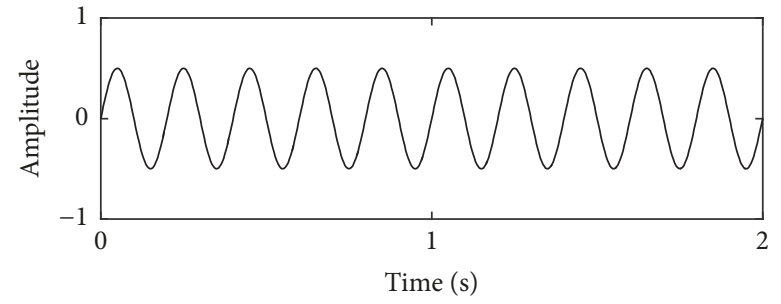

(b) $S_{\sin 2}$

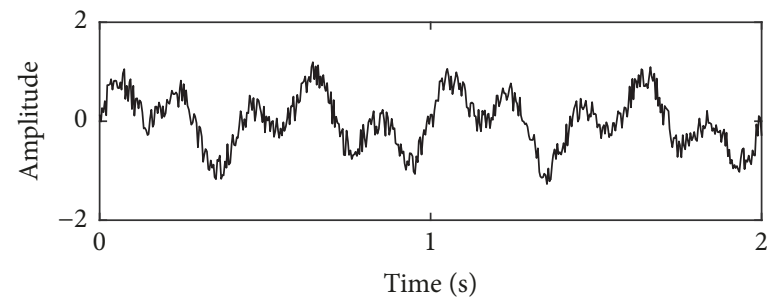

(c) $S_{c}$

FIGURE 8: Composite signal with two dominant frequencies.

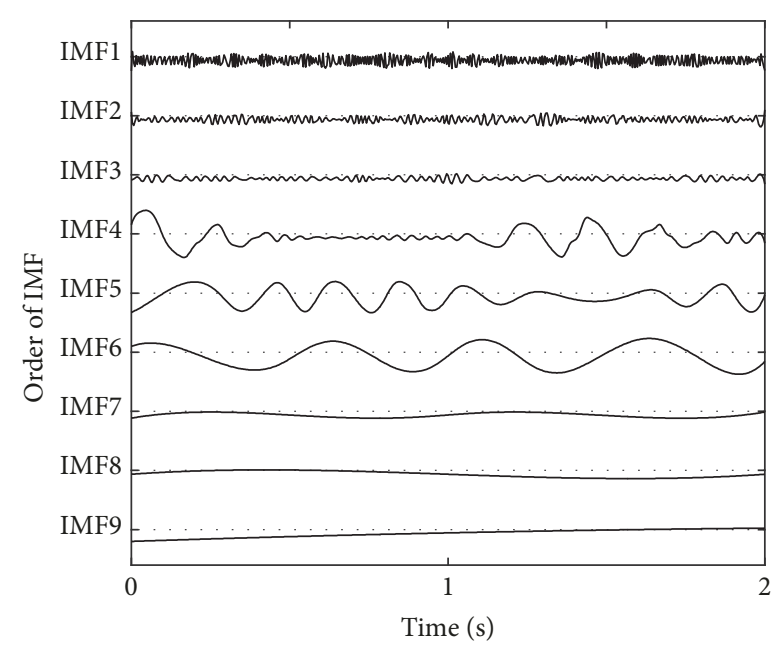

Figure 9: EMD results.

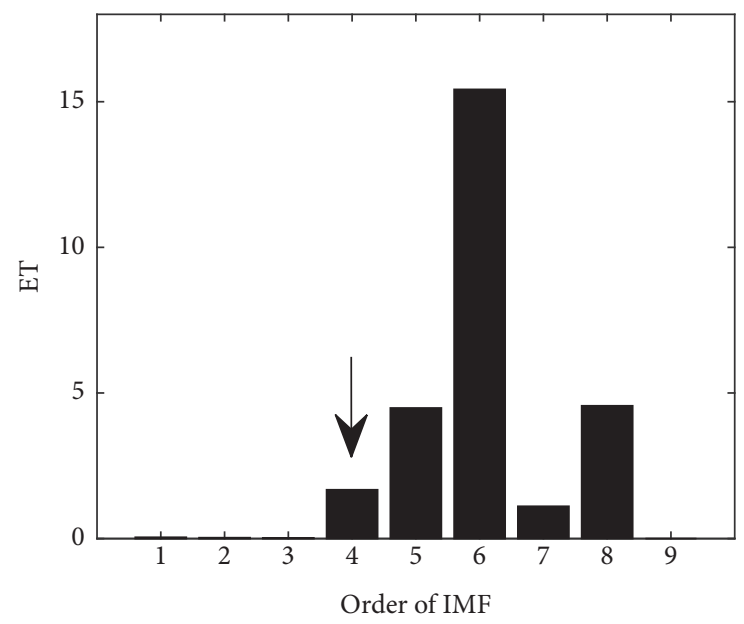

FIgURE 10: ET values of the IMFs.

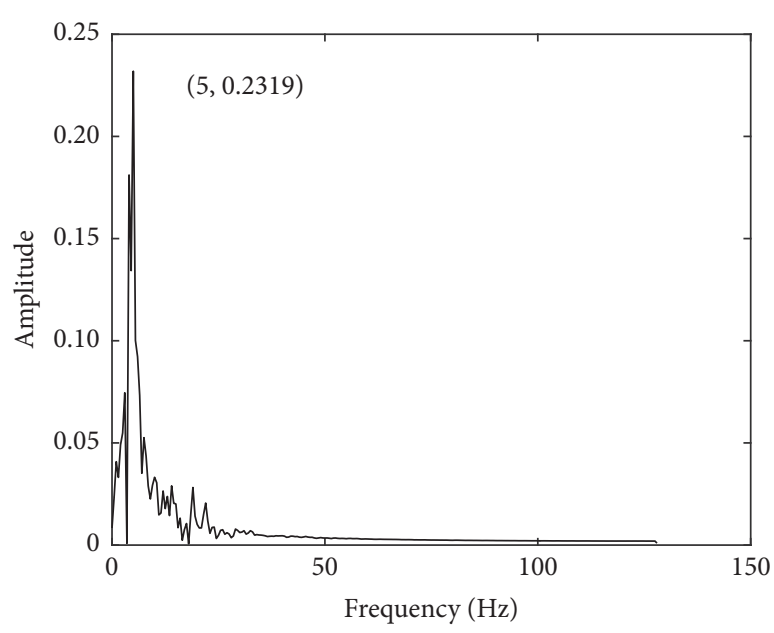

FIGURE 11: Auto-power spectrum of the $4^{\text {th }}$ IMF.

The main flows of MF-EEMD are as follows: (1) Decompose the original signal by traditional EMD or EEMD and determine the appropriate structuring element size based on the decomposition results. (2) Add white noise to the original signal and employ the MF to filter the polluted signal. (3) Adopt EMD to decompose the filtering signal. (4) Repeat the second and third processes by adding different series of white noise. (5) Make an ensemble of the numerous decomposition results and achieve the final decomposition results of EEMD. The above flows of MF-EEMD are shown in Figure 15, where the additional operations proposed in this paper are marked with a gray shade.

\section{Application Instance}

5.1. Analog Signal Decomposition. To verify the feasibility of the proposed method, the composite signal $S_{c}$ in Figure 8(c) 


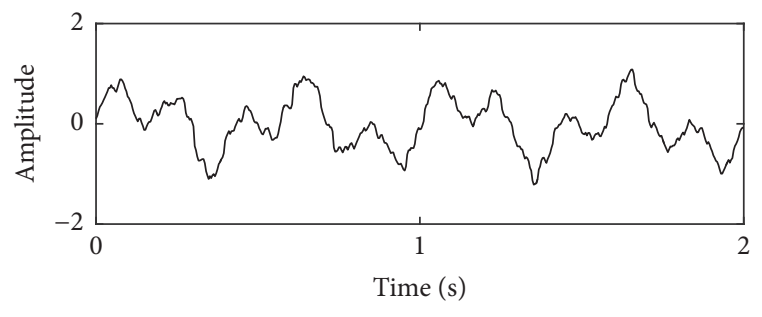

(a) $S_{M F}$

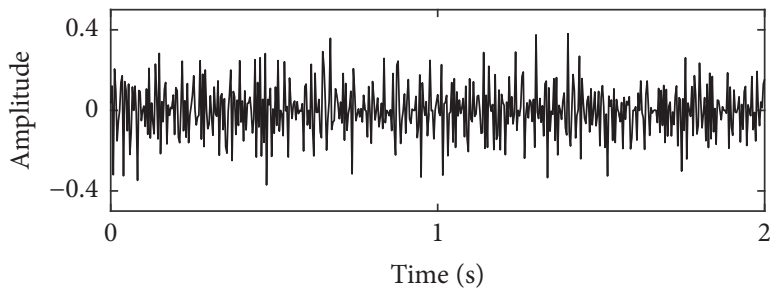

(b) Noise component

FIGURE 12: Filtering result of the MF.

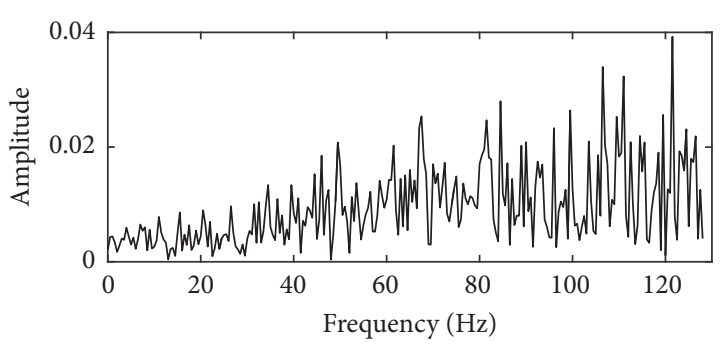

FIGURE 13: Fourier spectrum of the noise component.

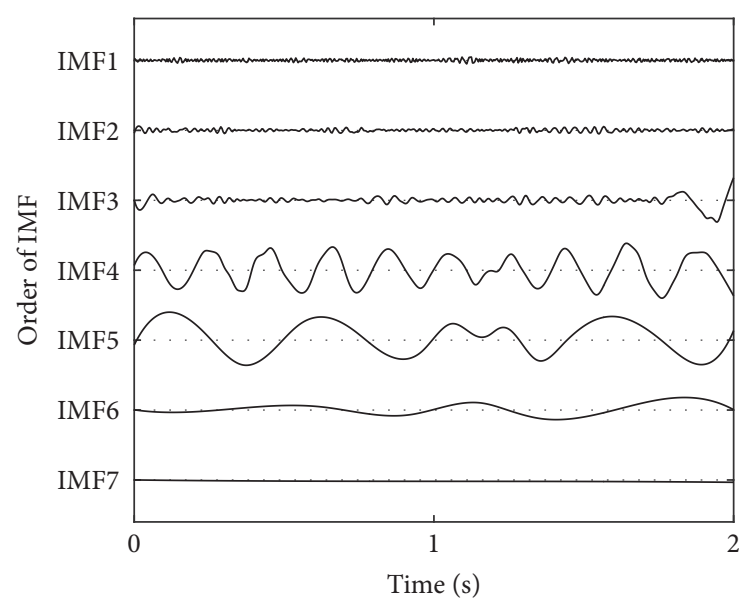

FIGURE 14: Results of the EMD combined with MF.

was selected again to be decomposed by EEMD and MFEEMD. During operations of the two methods, the level of white noise added in each operation of EMD was set as 0.1 times the standard deviation of $S_{c}$, and the number of ensembles was set as 500. The appropriate size of triangular structuring element was set as $W=3, H=0.1$. Nine IMFs are present in the traditional EEMD results, as shown in Figure 16, while there are 7 IMFs in the MF-EEMD results, as shown in Figure 17. The corresponding Hilbert spectra of the EEMD and MF-EEMD results are shown in Figures 18 and 19, respectively.

In Figure 16 , the $1^{\text {st }}$ - to $4^{\text {th }}$-order IMFs are noise, the $5^{\text {th }}$-order IMF is a $5 \mathrm{~Hz}$ component, the $6^{\text {th }}$-order IMF is a $2 \mathrm{~Hz}$ component, and the $7^{\text {th }}$ - to $9^{\text {th }}$-order IMFs are trends. However, the waveform of the $4^{\text {th }}$-order IMF from $1 \mathrm{~s}$ to $2 \mathrm{~s}$ is quite similar to that of the $5^{\text {th }}$-order IMF; there are slight mode mixing problems in the EEMD results. The mode mixing problems can also be observed in Figure 18, where there are disturbances around the $5 \mathrm{~Hz}$ spectrum.

In Figure 17, the $1^{\text {st }}$ - to $3^{\text {rd }}$-order IMFs are noise, the $4^{\text {th }}$ order IMF is a $5 \mathrm{~Hz}$ component, the $5^{\text {th }}$-order IMF is a $2 \mathrm{~Hz}$ component, and the $6^{\text {th }}$ - and $7^{\text {th }}$-order IMFs are trends. In Figure 19 , the $5 \mathrm{~Hz}$ and $2 \mathrm{~Hz}$ spectra are straight, and the background is quite clean. No obvious mode mixing problem is found in the MF-EEMD results.

5.2. Measured Signal Decomposition. For further verifying the proposed method, the acceleration signal measured from a shaking table test of a cable-stayed bridge model was taken as another instance. A photograph of the cable-stayed bridge model is shown in Figure 20. The input of the test was white noise with a peak value of $0.1 \mathrm{~g}$, and the input direction was lateral. A set of acceleration responses of the tower top with a sample frequency of $256 \mathrm{~Hz}$ was selected, as shown in Figure 21.

In this instance, the level of white noise added in each operation of EMD was set as 0.2 times the standard deviation of the measured signal, and the number of ensembles was set as 2,000. First, the measured signal was decomposed by EEMD, and 11 IMFs were obtained. The decomposition results along with the $E T$ values are shown in Figures 22 and 23. There is a sudden increase between the $3^{\text {rd }}$ and $4^{\text {th }} E T$ values, indicating that the desired $f$ is in the $4^{\text {th }}$-order IMF. The auto-power spectrum of the $4^{\text {th }}$-order IMF is shown in Figure 24, and the frequency of the peak point is $10.38 \mathrm{~Hz}$. The appropriate size of the structuring element was derived as $W=3$ and $H=0.3$ by (13) with $s f=256$ and $f=10.38$. Then, the measured signal was decomposed by MF-EEMD, and 9 IMFs were obtained, as shown in Figure 25.

To compare the mode mixing problems in detail, the local waveforms of the IMFs of the two methods are presented in Figures 26 and 27. Mode mixing problems are present in the $5^{\text {th }}$-order IMF of EEMD, and the waveforms marked with the red circle are similar to that of the $4^{\text {th }}$-order IMF. The waveforms marked with the blue circles are similar to that of the $6^{\text {th }}$-order IMF. However, there are no evident mode mixing problems in the IMFs of MF-EEMD.

The Hilbert spectra of the EEMD and MF-EEMD results are shown in Figures 28 and 29, respectively. In Figure 28, there are serious mode mixing problems in the two spectra of 


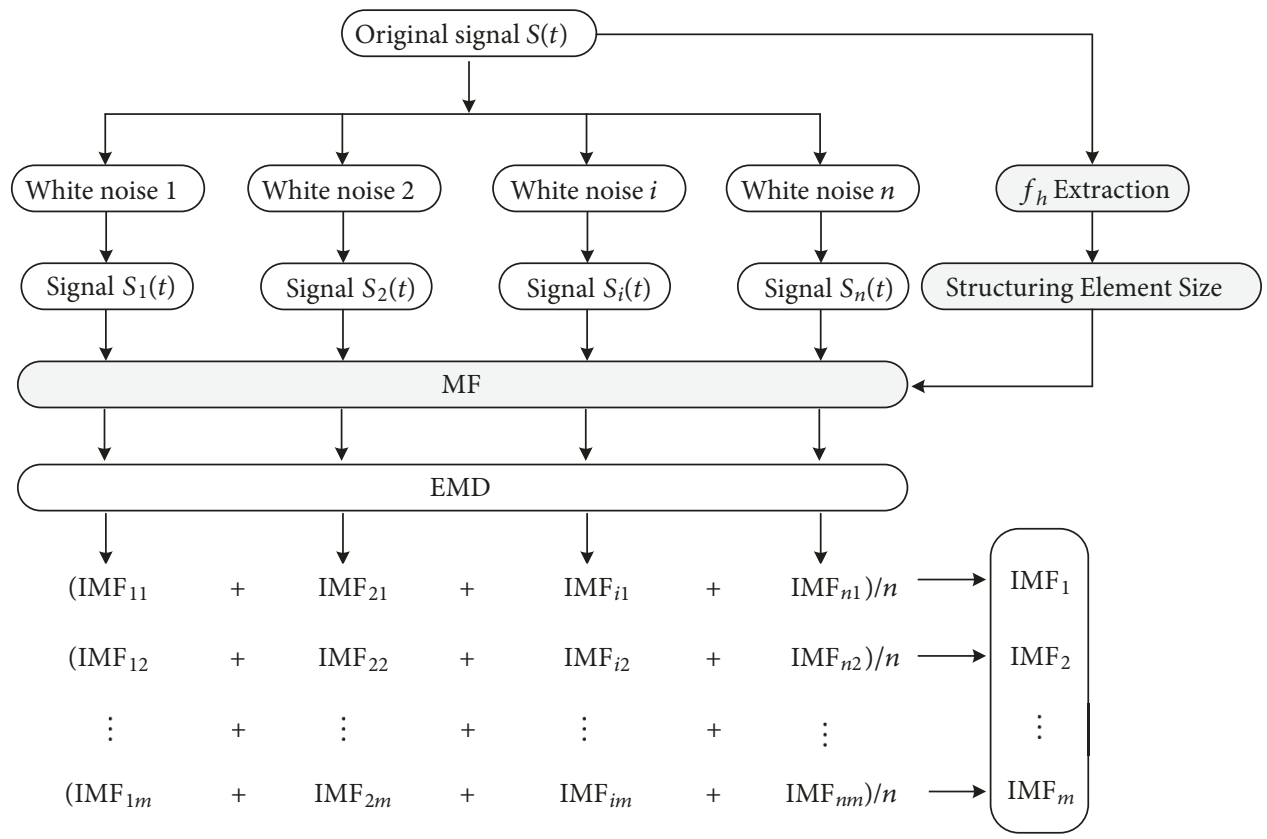

FIgURE 15: Operational flow of the MF-EEMD.

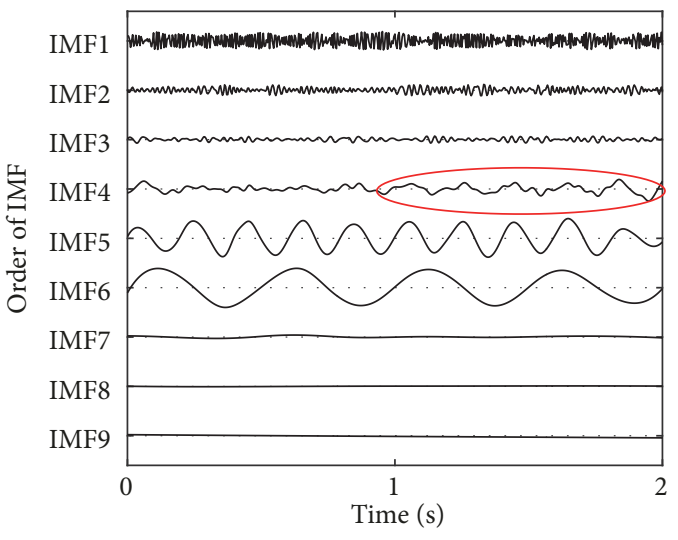

Figure 16: EEMD results.

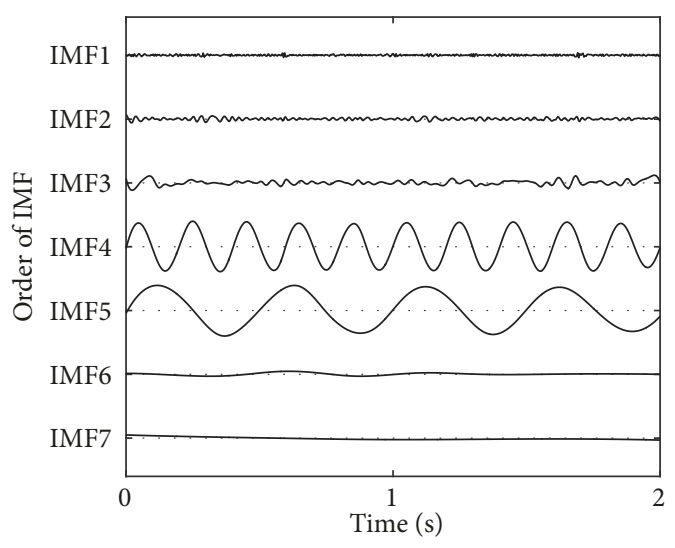

FIgURE 17: MF-EEMD results.

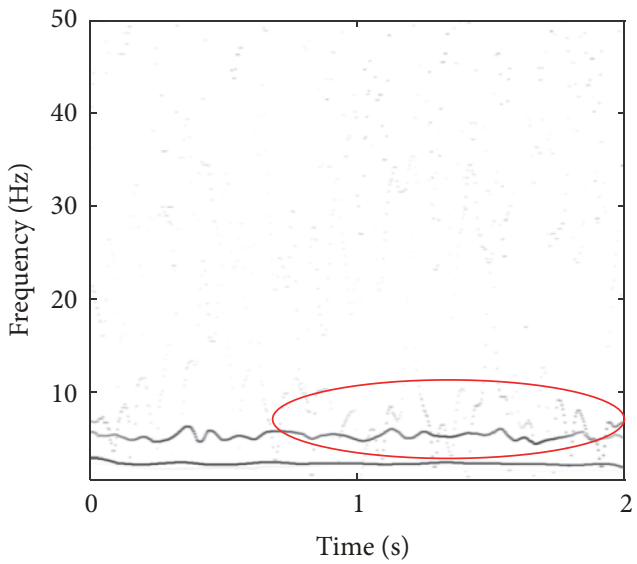

FIgURE 18: Hilbert spectrum of EEMD.

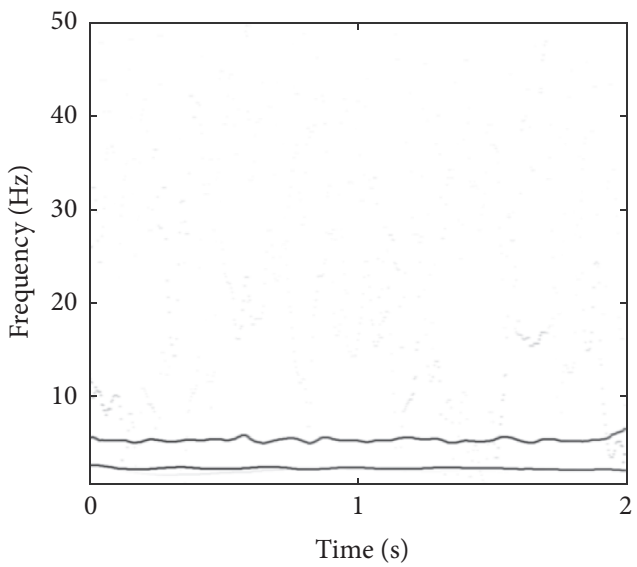

Figure 19: Hilbert spectrum of MF-EEMD. 


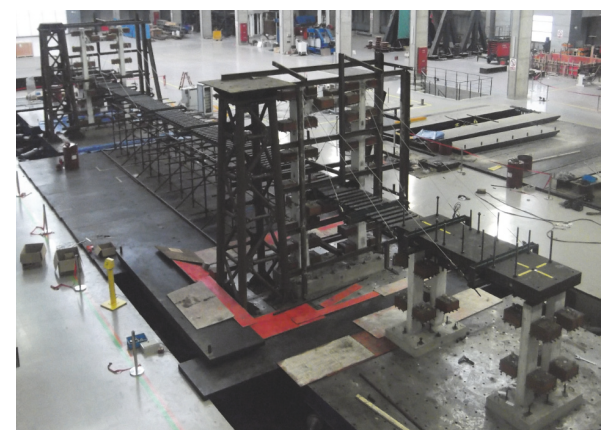

Figure 20: Cable-stayed bridge model.

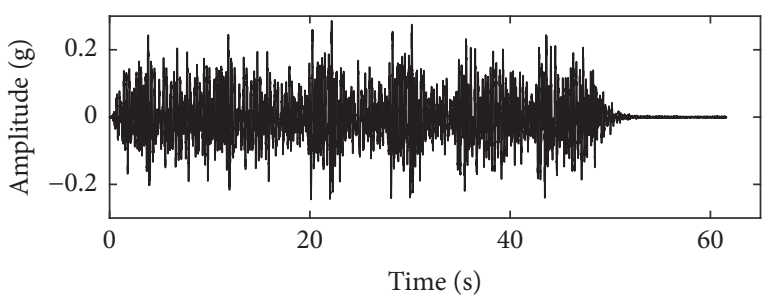

FIGURE 21: Acceleration response of the tower top.

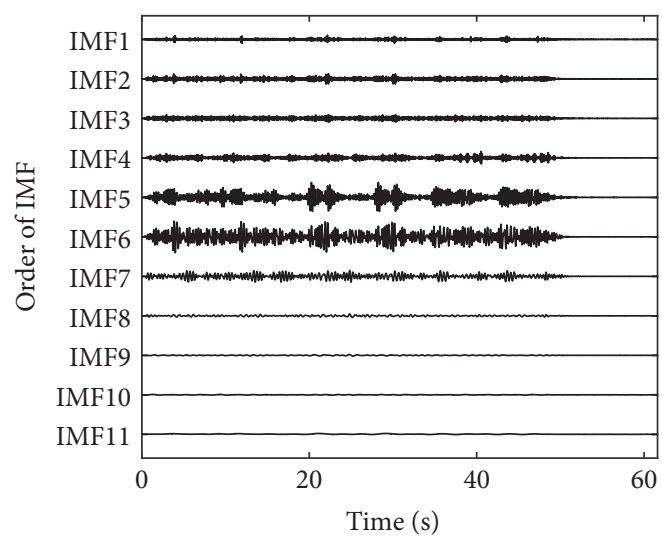

Figure 22: EEMD results.

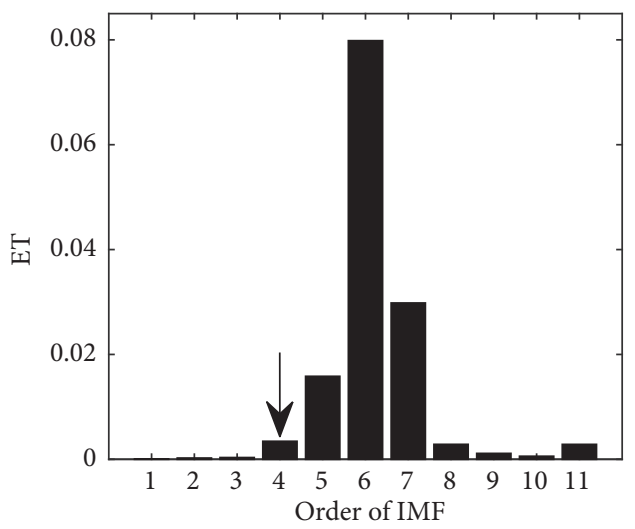

Figure 23: ET values of IMFs.

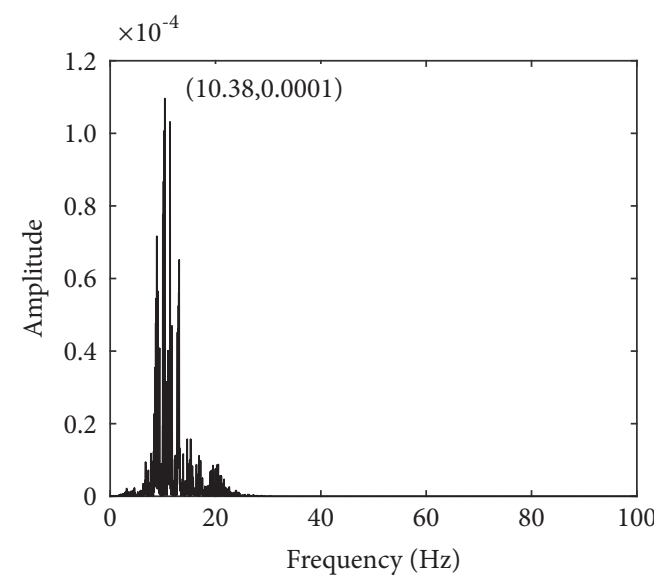

FIgURE 24: Auto-power spectrum of the $4^{\text {th }}$ IMF.

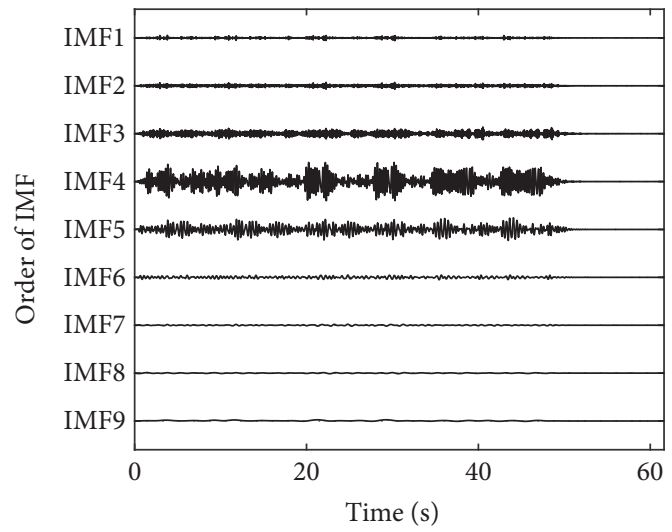

Figure 25: MF-EEMD results.

approximately $4 \mathrm{~Hz}$, and the fluctuations of their frequencies cannot be easily tracked. The spectrum of approximately $10 \mathrm{~Hz}$ is widely distributed in the frequency domain, which means that the corresponding waveforms of this spectrum are not smooth. In Figure 29, the mode mixing problems in the two spectra of approximately $4 \mathrm{~Hz}$ are mild, and the fluctuations of their frequencies are clear and easy to track. The distribution band of the spectrum of approximately $10 \mathrm{~Hz}$ is narrow, which means that the smoothness of its waveforms is improved. The comparison results show that the mode mixing problems in EEMD are effectively suppressed by MFEEMD.

\section{Conclusion}

MF-EEMD was proposed to suppress the mode mixing problems in EEMD. Structuring elements with different shapes and sizes were used in the MF to filter a series of analog signals. Based on the filtering results, the triangular shape was found to be superior to the linear and sinusoidal shapes, and an equation for calculating the appropriate sizes of the triangular structuring element was established. Then, the MF was used to filter the superfluous white noise components during the EMD operation. The MF could help suppress the mode 


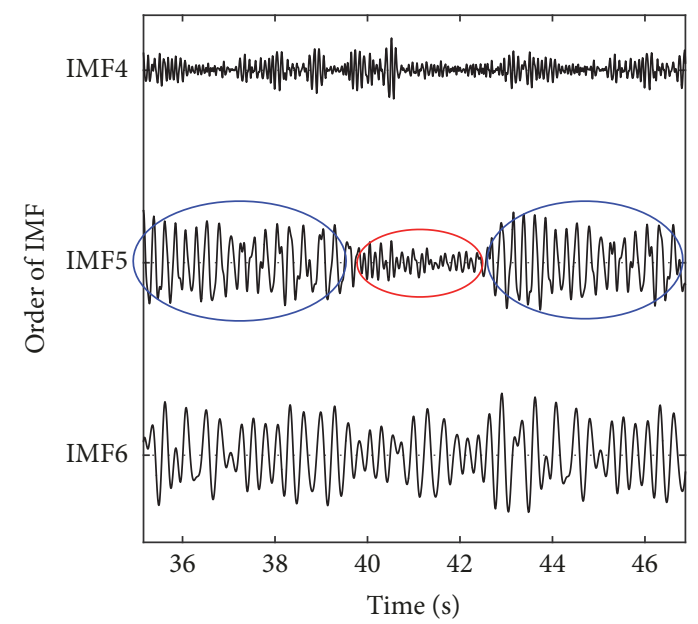

FIgURE 26: Local waveforms of the IMFs of EEMD.

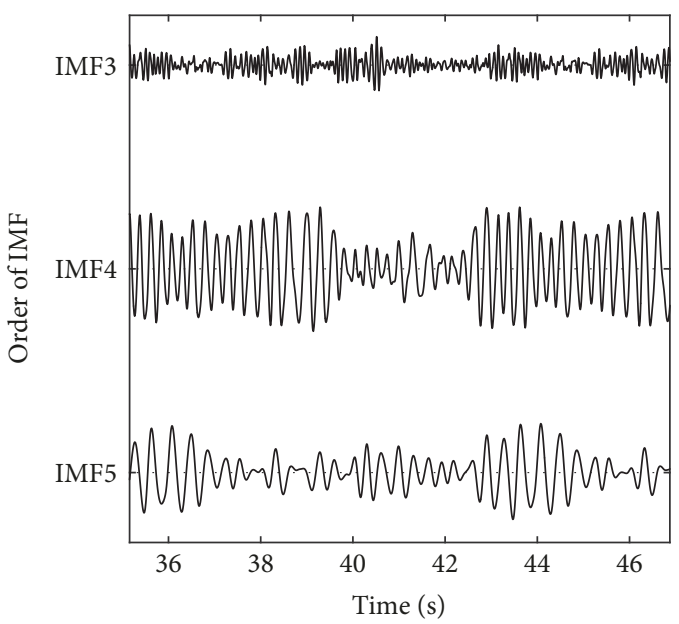

FIGURE 27: Local waveforms of the IMFs of MF-EEMD.

mixing problems and improve the decomposition efficiency. Based on the above results, an improved MF-EEMD method was proposed by combining the EEMD with MF. To verify the proposed method, an analog signal and a measured signal were decomposed by EEMD and MF-EEMD, respectively. The comparison results show that the mode mixing problems in EEMD are effectively suppressed by the MF-EEMD. Moreover, under the same stoppage criterion, fewer IMFs are archived by the MF-EEMD, which means that the proposed method also has a higher decomposition efficiency.

\section{Data Availability}

The data used to support the findings of this study are available from the corresponding author upon request.

\section{Conflicts of Interest}

The authors declare no conflicts of interest.

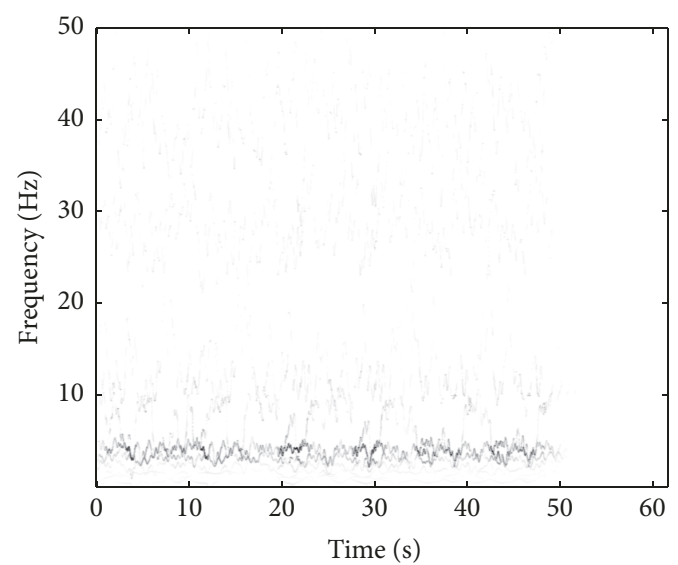

FIGURE 28: Hilbert spectrum of EEMD.

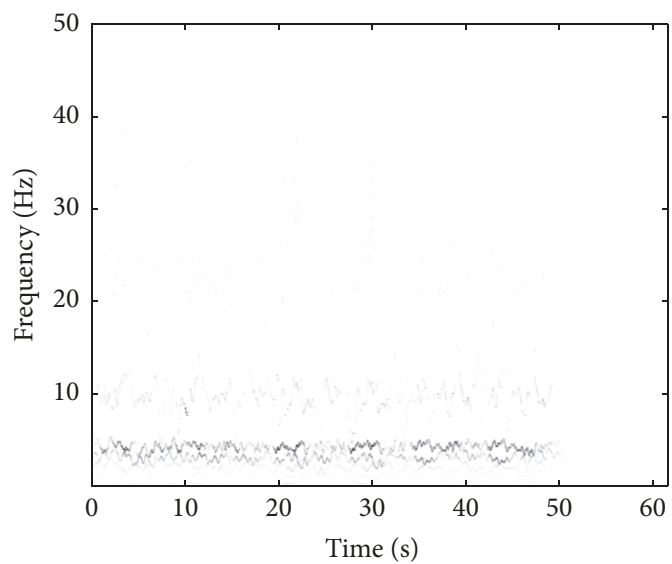

Figure 29: Hilbert spectrum of MF-EEMD.

\section{Acknowledgments}

This work was funded by the National Key R\&D Program of China (no. 2016YFC0802202), the National Natural Science Foundation of China (no. 51678489), and the Science and Technology Project of Power China (no. SCMQ-201728-ZB).

\section{References}

[1] C. R. Farrar and K. Worden, Structural Health Monitoring: A Machine Learning Perspective, Wiley, 2012.

[2] D. Pines and L. Salvino, "Structural health monitoring using empirical mode decomposition and the Hilbert phase," Journal of Sound and Vibration, vol. 294, no. 1-2, pp. 97-124, 2006.

[3] J. Vieira Filho, F. G. Baptista, and D. J. Inman, “Time-domain analysis of piezoelectric impedance-based structural health monitoring using multilevel wavelet decomposition," Mechanical Systems and Signal Processing, vol. 25, no. 5, pp. 1550-1558, 2011.

[4] R. N. Bracewell, The Fourier Transform and Its Applications, McGraw-Hill Book Company, 1986.

[5] N. E. Huang, Z. Shen, S. R. Long et al., "The empirical mode decomposition and the Hilbert spectrum for nonlinear and 
non-stationary time series analysis," Proceedings of the Royal Society of London A, vol. 454, no. 1971, pp. 903-995, 1998.

[6] C. R. Pinnegar and L. Mansinha, "Time-local Fourier analysis with a scalable, phase-modulated analyzing function: The Stransform with a complex window," Signal Processing, vol. 84, no. 7, pp. 1167-1176, 2004.

[7] U. Andreaus, P. Baragatti, P. Casini, and D. Iacoviello, "Experimental damage evaluation of open and fatigue cracks of multicracked beams by using wavelet transform of static response via image analysis," Structural Control and Health Monitoring, vol. 24, no. 4, pp. 1-16, 2017.

[8] N. E. Huang, M. L. C. Wu, S. R. Long et al., "A confidence limit for the empirical mode decomposition and Hilbert spectral analysis," Proceedings Mathematical Physical \& Engineering Sciences, vol. 459, no. 2037, pp. 2317-2345, 2003.

[9] B. Tang, S. Dong, and T. Song, "Method for eliminating mode mixing of empirical mode decomposition based on the revised blind source separation," Signal Processing, vol. 92, no. 1, pp. 248-258, 2012.

[10] Z. H. Wu and N. E. Huang, "Ensemble empirical mode decomposition: a noise-assisted data analysis method," Advances in Adaptive Data Analysis, vol. 1, no. 1, pp. 1-41, 2009.

[11] P. Flandrin, G. Rilling, and P. Gonçalvés, "Empirical mode decomposition as a filter bank," IEEE Signal Processing Letters, vol. 11, no. 2, pp. 112-114, 2004.

[12] M. E. Torres, M. A. Colominas, G. Schlotthauer, and P. Flandrin, "A complete ensemble empirical mode decomposition with adaptive noise," in Proceedings of the 36th IEEE International Conference on Acoustics, Speech, and Signal Processing, pp. 41444147, Prague, Czech Republic, May 2011.

[13] H. J. A. M. Heijmans, Morphological Filters, Springer, 2008.

[14] J. Saniie and M. A. Mohamed, "Ultrasonic flaw detection based on mathematical morphology," IEEE Transactions on Ultrasonics, Ferroelectrics and Frequency Control, vol. 41, no. 1, pp. 150160, 2002.

[15] Z.-F. Deng, Z.-P. Yin, and Y.-L. Xiong, "High probability impulse noise-removing algorithm based on mathematical morphology," IEEE Signal Processing Letters, vol. 14, no. 1, pp. 31-34, 2007.

[16] C. Yuan and Y. Li, "Switching median and morphological filter for impulse noise removal from digital images," Optik, vol. 126, no. 18, pp. 1598-1601, 2015.

[17] P. Lin, B. Chen, F. Cheng, and S. Huang, "A morphological mean filter for impulse noise removal," Journal of Display Technology, vol. 12, no. 4, pp. 344-350, 2016.

[18] S. Mukhopadhyay and B. Chanda, "An edge preserving noise smoothing technique using multiscale morphology," Signal Processing, vol. 82, no. 4, pp. 527-544, 2002.

[19] J. Serra, "Introduction to mathematical morphology," Computer Vision, Graphics, and Image Processing, vol. 35, no. 3, pp. 283305, 1986.

[20] J. Serra, Introduction of Morphological Filter in Image Analysis and Mathematical Morphology, Academic Press, 1988.

[21] H. Heijmans and C. Ronse, The Algebraic Basis of Mathematical Morphology: I. Dilations and Erosions, Academic Press Professional, 1990.

[22] C. Ronse and H. J. A. M. Heijmans, "The algebraic basis of mathematical morphology: II. Openings and closings," Computer Vision Graphics \& Image Processing, vol. 54, no. 1, pp. 7497, 1991.
[23] L. Smital, M. Vitek, J. Kozumplik, and I. Provaznik, "Adaptive wavelet wiener filtering of ECG signals," IEEE Transactions on Biomedical Engineering, vol. 60, no. 2, pp. 437-445, 2013.

[24] Z. H. Wu and N. E. Huang, "A study of the characteristics of white noise using the empirical mode decomposition method," Proceedings of the Royal Society A Mathematical, Physical and Engineering Sciences, vol. 460, no. 2046, pp. 1597-1611, 2004. 


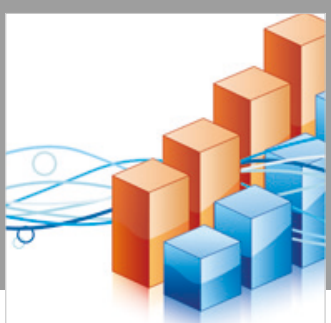

Advances in

Operations Research

\section{-n-m}
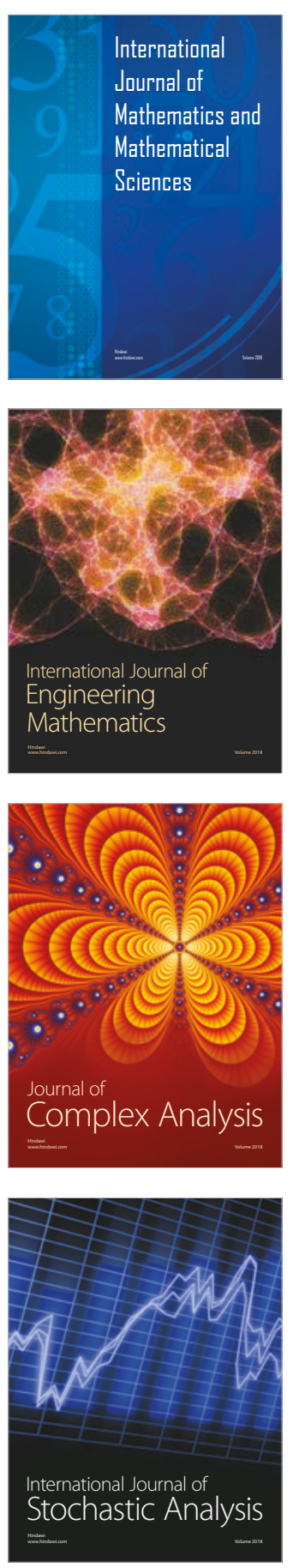
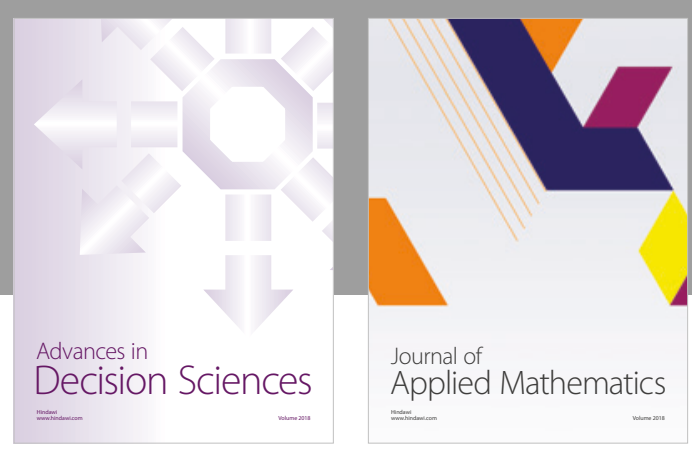

Journal of

Applied Mathematics
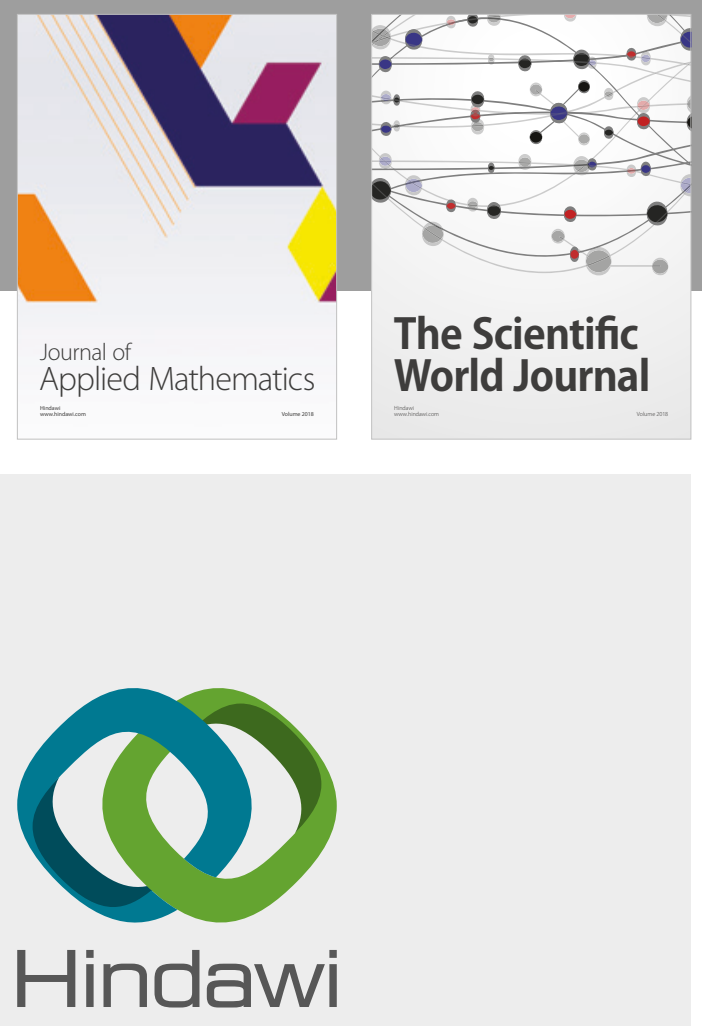

Submit your manuscripts at

www.hindawi.com

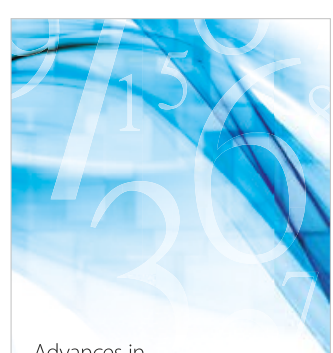

Advances in
Numerical Analysis
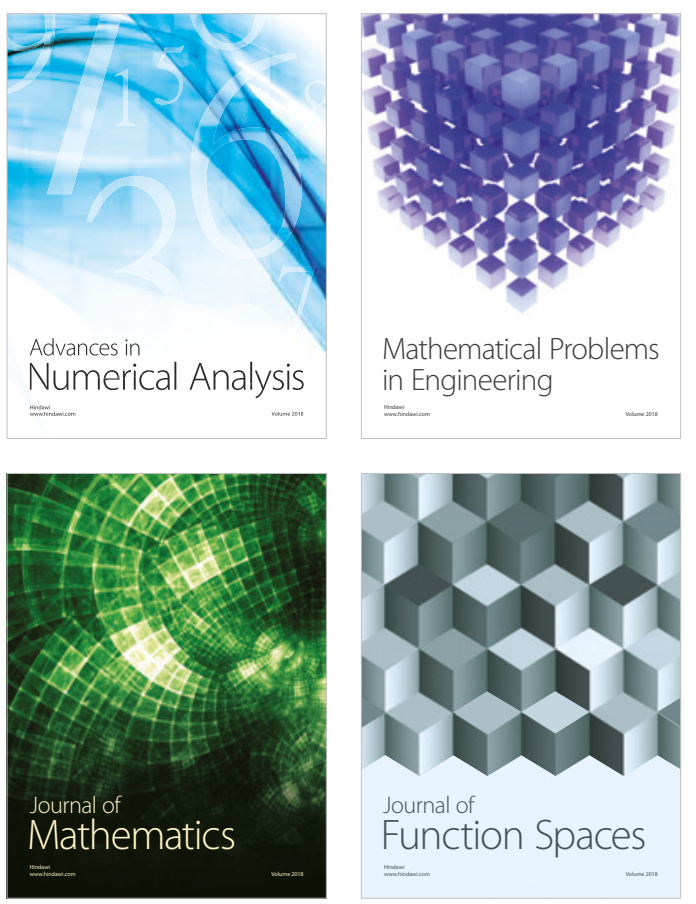

Mathematical Problems in Engineering

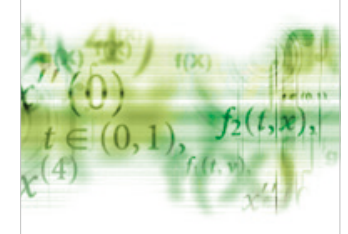

International Journal of

Differential Equations

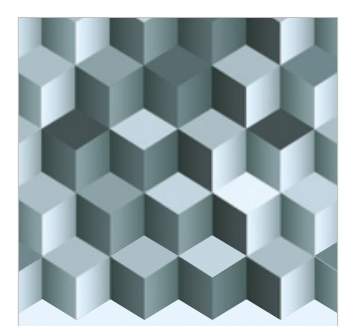

Journal of

Function Spaces

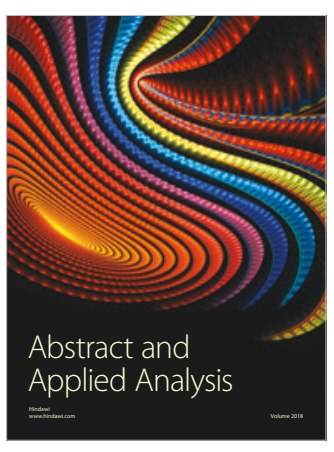

The Scientific

World Journal

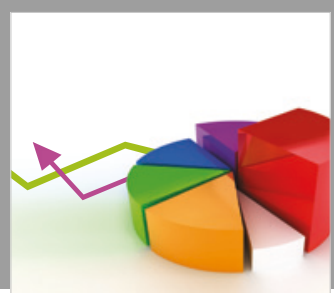

Journal of

Probability and Statistics
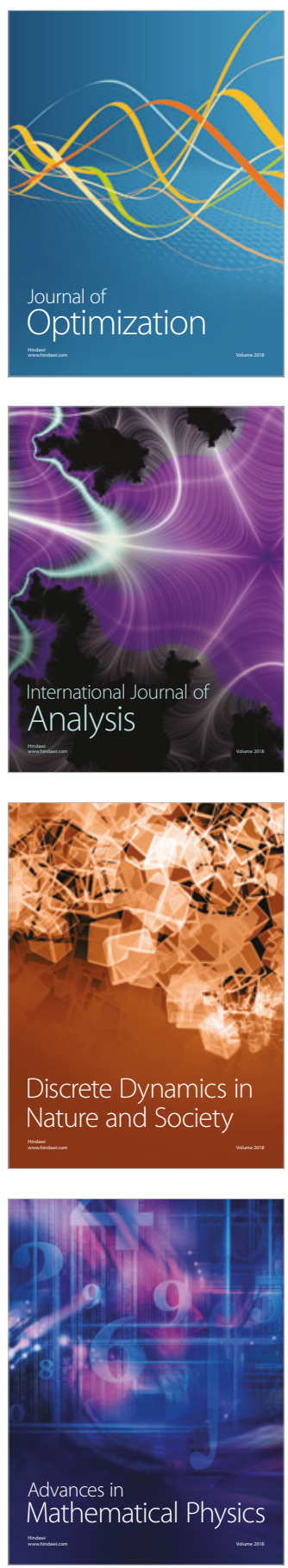